\title{
A Practical Fuzzy Adaptive Control Strategy for Multi-DOF Parallel Robot
}

\author{
Wei Meng ${ }^{1,2}$, Zude Zhou ${ }^{1,2}$, Quan Liu ${ }^{1,2}$, Qingsong Ai ${ }^{1,2}$ \\ 1 School of Information Engineering, Wuhan University of Technology, 122 Luoshi Road, Hongshan District \\ Wuhan, Hubei 430070, China \\ 2 Key Lab. of Fiber Optic Sensing Technology and Information Processing, Wuhan University of Technology, Ministry of \\ Education, 122 Luoshi Road, Hongshan District, Wuhan, Hubei 430070, China \\ e-mail: weimeng@whut.edu.cn
}

\begin{abstract}
Multiple Degrees of Freedom (DOF) parallel robots possess the advantages of being compact structure, great stiffness, stability and high accuracy, so such platforms have been widely used in application areas as diverse as the spacecraft motion simulators, radio telescopes, and medical rehabilitation devices. In this paper, after giving a brief review on the control strategies for parallel robot, a 6-DOF robot system for medical purposes based on simulation as well as real environment is established. In order to improve the position tracking accuracy for such objects with time-varying and nonlinear parameters, a practical fuzzy adaptive controller is designed based on the kinematics of parallel platform, where fuzzy inference units are utilized to modify the PID parameters in real-time by using the position feedback from the robot actuators. Finally, both virtual and actual experiment results demonstrate that the proposed algorithm is able to effectively reduce the position tracking errors compared with the traditional PID controller, and the reliability and feasibility of such parallel robotic system can also be guaranteed.
\end{abstract}

Keywords-Parallel robot; Stewart platform; fuzzy adaptive algorithm; control strategy

\section{INTRODUCTION}

Medical rehabilitation robot is widely believed to be an interdisciplinary research area which become an outstanding field attracting more and more attentions from both industrial and academic domains[1]. Applying robot to rehabilitation not only be able to release physicians from the heavy burden of training mission, but also provide a platform to evaluate the convalescence results by analyzing data recorded during the training process[2]. The mechanical design of the robot is the basis of robot-assisted rehabilitation system, which should comply with the disciplines of simple, lightweight, as well as easy controlling. 6-DOF parallel robot is a feasible solution for its simple structure and superior adaptability, enabling system to be adjustable [3]. Moreover, the Stewart platform is a typical parallel robot with six degrees of freedom. It is nowadays used various fields where with strict constrains, such as aviation (flight simulators), industry (manufacturing) and medicine (long bone surgery) [4].

As a multi-input and multi-output (MIMO) and nonlinear system, 6-DOF parallel robot's position and orientation control are results interaction and coordination of six actuators. Wu et al. presented an adaptive control strategy based on $\mathrm{H} \infty$ robust algorithm to overcome the adverse effects caused by parameter variations and disturbances[5].
Li et al. proposed an integral variable structure controller to enhance robustness for interference and perturbation[6]. Besides, many literatures tend to propose control approaches based on simulation, but not verified by experiments in practical environment[7]. Meanwhile, fuzzy control method is probably an effective approach to control such objects with time-varying and nonlinear parameters for its advantages of independent on mathematical model, as well as good robustness and nonlinear characteristics.

In this paper, a multi-DOF parallel robot based on Stewart platform for lower limb rehabilitation is presented. After studying its kinematics and mathematical models in Section II, a high-precision position control strategy based on fuzzy adaptive algorithm is proposed in Section III. In order to improve the trajectory tracking accuracy, position feedback information was utilized to modify the adaptive parameters in real-time through fuzzy logic inference units. While in Section IV, experiments based on SimMechanics and real robot system were conducted, and finally, the results are analyzed and the conclusions are drawn in Section V.

\section{INVERSE KINEMATICS}

The Stewart platform is a parallel robot whose operation is based on the simultaneous movement of its six-axis. The mechanical structure of Stewart consists of the moveable upper platform, the fixed based platform, and six ball screw actuators. Servo motors drive six electric cylinders to change the length of each leg, and thus achieve the desired trajectory of the moving platform. The kinematics problems of parallel robot can be divided into the inverse kinematics and the forward kinematics[8], where the inverse ones means calculate the joint coordinates of six legs when the position of the end effector is known, while forward kinematics vice versa. If given the desired translational and rotational motion of the upper platform, the inverse kinematics could be used to transform the trajectory into the displacement of each actuator, and thus the respective joints are able to be controlled instead of the moving platform. The geometric model of a Stewart platform and its victor diagram can be seen in Fig. 1, where radius of the upper platform is defined as $r_{b}$, and the angle is $\theta_{1}$, likewise, the parameters of the fixed platform are defined as $r_{a}$ and $\theta_{2}$, respectively. 


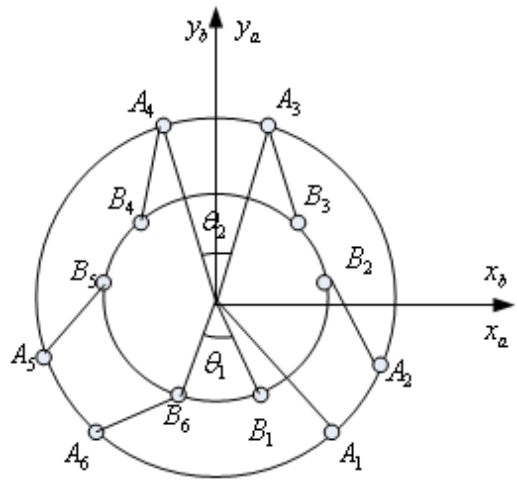

Figure 1. Geometric model and victor diagram of the platform.

A generalized position vector $q$ can be got, whose elements are the six variables chosen to describe the translation and orientation, which defined as equation (1), while the joint space coordinate vector $l$ is defined as (2)

$$
\begin{aligned}
& q=[x, y, z, \alpha, \beta, \gamma]^{T} . \\
& l=\left[l_{1}, l_{2}, l_{3}, l_{4}, l_{5}, l_{6}\right]^{T} .
\end{aligned}
$$

Defining vector $B_{i}$ describes the position of $i$-th upper vertices with respect to upper platform coordinate system and $A_{i}$ describes the position of the $i$-th base vertices with respect to the base coordinate system.

$$
\begin{aligned}
& B_{i}=\left[\begin{array}{llll}
r_{b} \cos \lambda_{i} & r_{b} \sin \lambda_{i} & 0
\end{array}\right]^{T} . \\
& A_{i}=\left[\begin{array}{llll}
r_{a} \cos \varphi_{i} & r_{a} \sin \varphi_{i} & 0
\end{array}\right]^{T} .
\end{aligned}
$$

The translation can be denoted as $T=[x, y, z]^{T}$, and the can be described by the orientation matrix rot which can be written as equation (5). The rotation matrix that around each axis can be expressed as following:

$$
R o t=R_{\alpha} R_{\beta} R_{\gamma}=\left[\begin{array}{ccc}
c \alpha \cdot c \beta & c \alpha \cdot s \beta \cdot s \gamma-s \alpha \cdot c \gamma & c \alpha \cdot s \beta \cdot c \gamma+s \alpha \cdot s \gamma \\
s \alpha \cdot c \beta & s \alpha \cdot s \beta \cdot s \gamma+c \alpha \cdot c \gamma & s \alpha \cdot s \beta \cdot c \gamma-c \alpha \cdot s \gamma \\
-s \beta & c \beta \cdot s \gamma & c \beta \cdot c \gamma
\end{array}\right]
$$

Finally, the expression of the length vector $l_{i}$ of leg $i$ as difference between $B_{i}$ and $A_{i}$ coordinates:

$$
l_{i}=\left\|T+\operatorname{Rot} \cdot B_{i}-A_{i}\right\|, \quad(i=1,2, \cdots, 6) .
$$

Then relationship between $l=\left[l_{1}, l_{2}, l_{3}, l_{4}, l_{5}, l_{6}\right]^{T}$ and $q=[x, y, z, \alpha, \beta, \gamma]^{T}$ is possible to be obtained. Based on the inverse kinematics of the Stewart, the displacement vector and length of each leg can be calculated if given the desired position and posture of the moving platform.

\section{THE FuZZY ADAPTIVE CONTROLLER}

The purpose of the fuzzy adaptive controller is to find out the relationship based on fuzzy rules between the three parameters of PID controller $\left(K_{p}, K_{i}, K_{d}\right)$ and the position error of each leg. In this system, the PID parameters can be adjusted in real-time based on the position error $e$ and differential ec. In this way the controller parameters are able to be tuned to meet the requirements of different position and orientation control, so that the controller would have a good dynamic and static performance. In order to achieve the smooth control of the parallel robot, the designed position close-loop controller consists of a fuzzy logic controller as well as a PID controller. Based on the position data feedback recorded by encoder sensors embedded in the actuators, the position error and its rate of change are used as the input of the fuzzy controller, and the de-fuzzified outputs are used for adjusting the PID parameters. Fig. 2 shows the diagram of proposed fuzzy adaptive control system.

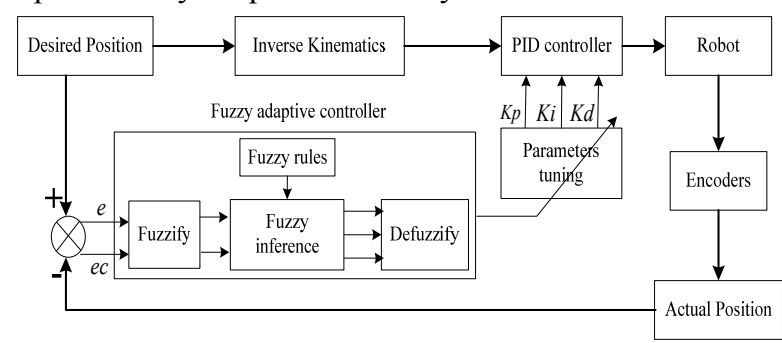

Figure 2. The proposed fuzzy adaptive controller.

The fuzzy rules can be determined by the previous expert experience and knowledge base of the relationship between PID parameters and the position errors. Based on the parameters tuning principle, the output variables $\Delta K_{p}, \Delta K_{i}$, $\triangle K_{d}$ can be adjusted automatically. In this control system, Mamdani's inference rules are adopted as a fuzzy set $\mathrm{R}$ (relationship) expressing fuzzy statement, and the inference process is shown as follows in Fig. 3.

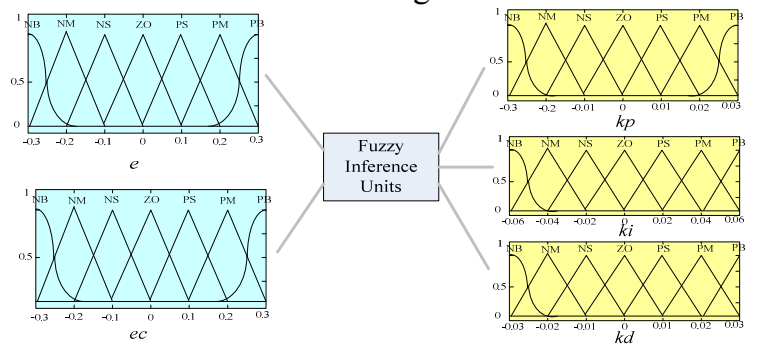

Figure 3. Fuzzy inference process.

According to the fuzzy subsets and assignment rules, as well as the control mode of each parameter, the fuzzy matrix of PID parameters can be obtained by fuzzy reasoning and inferring, and then corrected parameters can be calculated based on the initial value of the PID parameters, and thus achieve the tuned parameter values for robot controller. 


\section{EXPERIMENT SETUP}

\section{A. Simulation}

For simulation experiment, an implemented Stewart platform design template which consists in six main blocks corresponding to the six legs of the platform is used. Each of these entrance hangs a force and not a PWM signal for obvious reasons of simplicity, as shown in Fig. 4. In this part, a comparatively independent control over each actuator is developed, in order to find a method to actuate the six leg forces to properly position the mobile plate of the Stewart Platform when given a desired trajectory. Initially, the desired position and orientation of the mobile plate with respect to the fixed base plate by demand are given, with

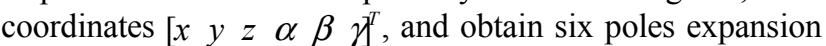
displacements through a conversion step from classical coordinates to leg length, according to platform's kinematics.
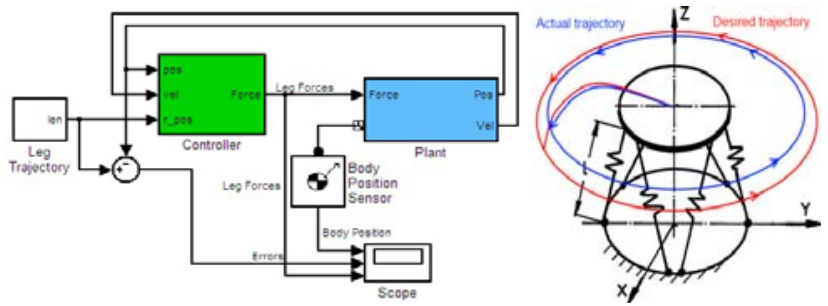

Figure 4. Global simulation and the desired trajectory.

In order to quantify the controllers for different platform speed looking at simulated trajectory accuracy, both the desired and actual trajectories are measured and analyzed. In the rehabilitation robot applications, the desired trajectory of the plate can be decomposed into a straight-line and an arc trajectory, and here a round arc trajectory as shown in Fig. 4 is provided to conduct experiments. According to predefined trajectory, the desired length and speed of each actuator can be obtained through the inverse kinematics, driving each actuator to reach the defined displacement enables the upper platform to reach the desired trajectory. From Fig. 5 and Fig. 6 we observe the position tracking error as the results of PID controller and the proposed fuzzy controller.

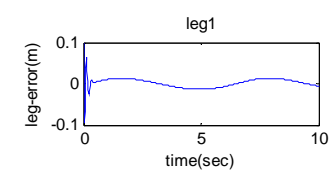

leg3

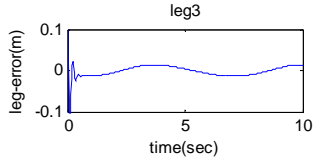

leg5
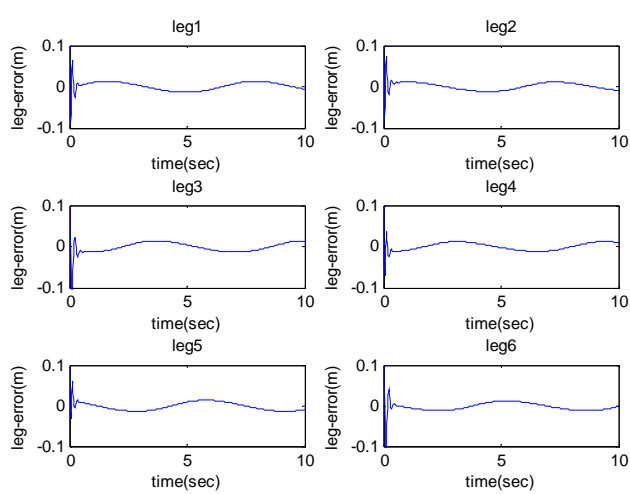

$\operatorname{leg} 4$

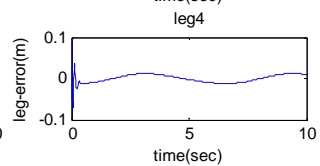

leg6

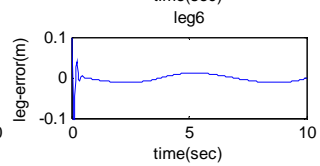

Figure 5. Position tracking error of PID controlled robot (simulation).
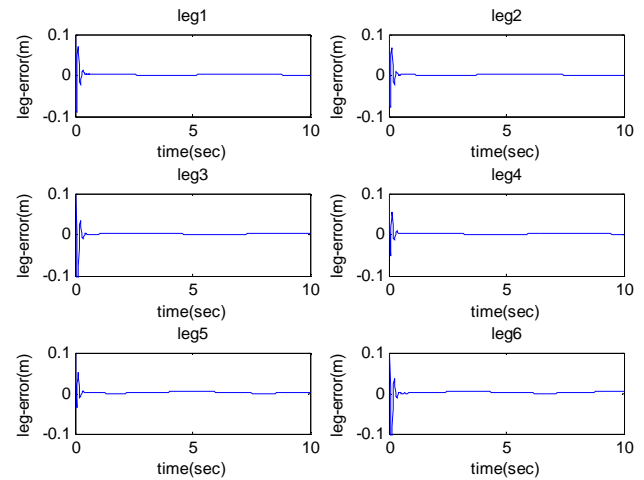

Figure 6. Position tracking error of fuzzy controlled robot (simulation).

The classical PID controller seems to be satisfactory only for the low speed applications, but as the speed increases, the position error becomes too significant and can not meet anymore the main objectives: accuracy. The use of a new controller is therefore required. The simulation results show that the fuzzy adaptive controller is able to make full use of the advantages of the PID controller and the fuzzy controller, for greatly improving the tracking performance compared with that of traditional ones.

\section{B. Practical applications}

The Stewart platform in real environment is designed as shown in Fig. 7: the set consists of a low-level control unit, connecting the platform itself to a computer which provides the high-level control. The six joints of the parallel robot are driven by six Panasonic Minas motor drivers, with the output power of $400 \mathrm{~W}$ and rated speed of $3000 \mathrm{r} / \mathrm{min}$, where the incremental photoelectric encoders are mounted coaxially with servo motor to provide position feedback.

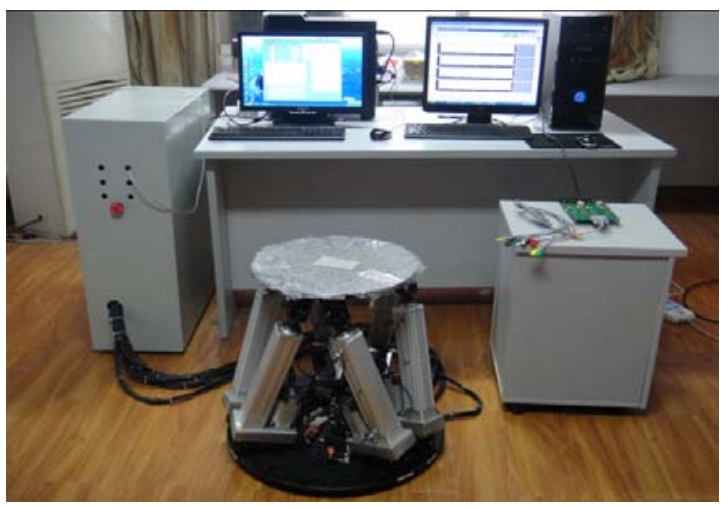

Figure 7. The Stewart platform setup.

In this experimental system, the radius of upper moving plate is $180 \mathrm{~mm}$, while the radius of the fixed base plate is $270 \mathrm{~mm}$, respectively. The trajectory for upper platform is defined as following: the initial position of the platform center is $(0,0,0)$, and the plate is then moved to $(90,0,90)$, here the unit is $\mathrm{mm}$, which is followed by moving a counterclockwise circular and turning $\pi$ rad. Fig. 8 and Fig. 9 give 
the position tracking error as the results of PID controller and the proposed fuzzy controller in real Stewart platform, where data are measured by photoelectric encoders.
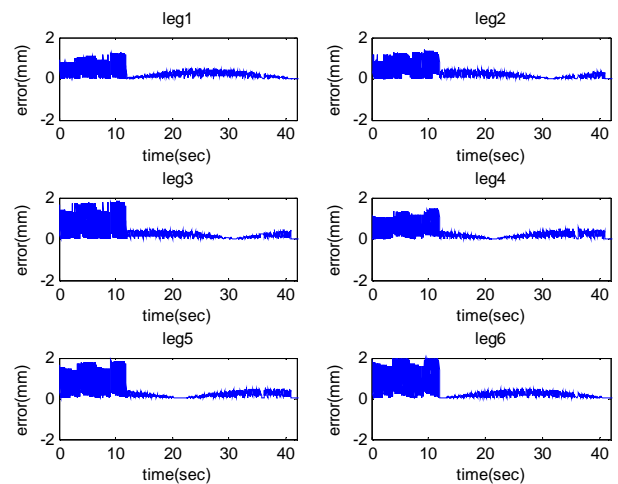

Figure 8. Position tracking error of PID controlled robot (real robot).
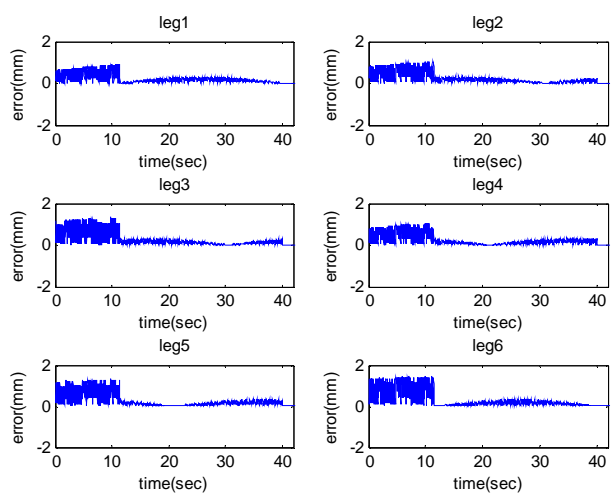

Figure 9. Position tracking error of fuzzy controlled robot (real robot).

Based on the analysis of experimental results above, it is obvious that the control strategy developed in this paper can follow the initial desired trajectory in a good performance. Compared with the PID controller, the proposed controller effectively reduces the position tracking delay and dynamic deviation, and the parallel robot operated more smoothly under a fuzzy controller. In other words, the designed fuzzy controller can be used in various applications for its ability to obtain efficient and reliable robot motion control as long as the defined trajectory is within industrial constraints, and this in turn, provides a feasible solution for position control of rehabilitation parallel robots.

\section{CONCLUSIONS}

It is always of great significance to maintain the tracking accuracy and stability of position control for rehabilitation applications[9]. An effective control system for Stewart robot used for lower limb rehabilitation is designed in this paper. Based on the study on parallel robot's kinematics, a prototype robotic system and control architecture of a 6-DOF parallel robot is established. Through the establishment of robot's prototype, a fuzzy adaptive controller is developed, and the classical PID controller is also tested on integrated simulation system based on Matlab and the real experimental platform designed. The experiment results demonstrate that applying the proposed adaptive fuzzy control method in 6DOF parallel robot manipulator is reasonable and valid, ensuring the tracking accuracy of the position and improving the stability and robustness of the system.

Currently, it seems there are still not enough realistic applications of parallel robots, thus it is worthwhile to propose a fuzzy adaptive controller that can achieve a good performance in the actual control for promoting the progress of related research. While the future works could involve improving dynamic response of such parallel platform and chasing for a better trajectory tracking performance by combining the fuzzy controller with other advanced control techniques, such as artificial neural network and sliding mode control strategies. Furthermore, in order to improve the rehabilitation results, force feedback between the robot and the patient could be taken into consideration, putting the position/force hybrid control and impedance control into practice is also important in this field.

\section{ACKNOWLEDGMENT}

This work is supported by the National High Technology Research and Development Program ("863" Program) of China under Grant no. 2012AA041203.

\section{REFERENCES}

[1] H. S. Lo, S. Q. Xie, "Exoskeleton robots for upper-limb rehabilitation: State of the art and future prospects," Medical Engineering \& Physics, 2011, pp. 1-8.

[2] R. Colombo, F. Pisano, S. Micera, and et al, "Robotic techniques for upper limb evaluation and rehabilitation of stroke patients," IEEE Transactions on Neural Systems and rehabilitation engineering, vol. 13, no. 3, 2005, pp. 311-324.

[3] S. Hussain, S. Q. Xie, G. Liu, "Robot assisted treadmill training: Mechanisms and training strategies," Medical Engineering \& Physics, vol. 33, 2011, pp. 527-533.

[4] Y. H. Tsoi, S. Q. Xie, A. E. Graham, "Design, modeling and control of an ankle rehabilitation robot," Design and Control of Intel. Robotic Systems, SCI vol. 177, 2009, pp. 377-399.

[5] B. Wu, S. Wu, K. Zhao, "Current status and development tendence of Stewart platform control strategy," Machine Tool \& Hydraulics, vol. 10, 2005, pp. 5-9.

[6] Y. Li, Y. Wang, Z. Chen, et al. "The state of research on intelligent control of parallel robot," Machine Tool \& Hydraulics, vol. 36, no.12, 2008, pp. 180-183.

[7] H. Guo, Y. Liu, G. Liu, et al, "Cascade control of a hydraulically driven 6-DOF parallel robot manipulator based on a sliding mode," Control Engineering Practice, vol. 16, 2008, pp. 1055-1068.

[8] P. K. Jamwal, S. Q. Xie, Y. H. Tsoi, and et al, "Forward kinematics modeling of a parallel ankle rehabilitation robot using modified fuzzy inference," Mechanism and Machine Theory, vol. 45, 2010, pp. 15371554 .

[9] D. Bradley, M C. Acosta, M. Hawley, et al., "NeXOS - The design, development and evaluation of a rehabilitation system for the lower limbs," Mechatronics, vol. 19, 2009, pp. 247-257. 